\title{
Accuracy of Intra-oral Radiography and Cone-beam CT in the Diagnosis of Buccal Bone Loss.
}

\section{Veronique Christiaens}

Ghent University

\section{Ruben Pauwels}

Aarhus University

\section{Bassant Mowafey}

KU Leuven

Reinhilde Jacobs ( $\nabla$ reinhilde.jacobs@kuleuven.be )

KU Leuven

\section{Research Article}

Keywords: Cone Beam Computed Tomography (CBCT), dentistry, maxillofacial field, Computed Tomography (CT) scan,intra-oral radiography (IOR)

Posted Date: February 16th, 2021

DOl: https://doi.org/10.21203/rs.3.rs-196788/v1

License: (c) (1) This work is licensed under a Creative Commons Attribution 4.0 International License. Read Full License 


\section{Abstract}

\section{Background}

The use of Cone Beam Computed Tomography (CBCT) in dentistry started in the maxillofacial field, where it was used for complex and comprehensive treatment planning. Due to the reduced radiation dose compared to a Computed Tomography (CT) scan, СBCT has become a frequently used diagnostic tool in dental practice. However, published data on the accuracy of CBCT in the diagnosis of the buccal bone level is lacking. The aim of this study was to compare the accuracy of intra-oral radiography (IOR) as well as CBCT in the diagnosis of the extent of buccal bone loss.

\section{Methods}

A dry skull was used to create a buccal bone defect at the most coronal level of a first premolar; the defect was enlarged apically in steps of $1 \mathrm{~mm}$. After each step, IOR and CBCT were taken. Based on CBCT data 2 observers jointly selected 3 axial slices at different levels of the buccal bone, as well as one transverse slice. Six dentists participated in radiographic observations. First, all observers received the 10 intra-oral radiographs and each observer was asked to rank the intra-oral radiographs on the extent of the buccal bone defect. Afterwards, the procedure was repeated with the CBCTs based on a combination of axial and transverse information. For the second part of the study, each observer was asked to evaluate axial as well as transverse CBCT slices on the presence or absence of a buccal bone defect.

\section{Results}

The percentage of buccal bone defect progression rankings that were within 1 of the true rank was $32 \%$ for IOR and $42 \%$ for CBCT. On average, kappa values were increased by 0.384 for СBCT compared with intra-oral radiography. The overall sensitivity and specificity of CBCT in the diagnosis of the presence or absence of a buccal bone defect was 0.89 and 0.85 , respectively. The average area under the curve (AUC) of the ROC was 0.892 for all observers.

\section{Conclusion}

When CBCT images are available for justified indications, other than bone level assessment, such 3D images are more accurate and thus preferred to $2 \mathrm{D}$ images to assess periodontal buccal bone. For other clinical applications, intra-oral radiography remains the standard method for radiographic evaluation.

\section{Introduction}

Almost every diagnosis in dentistry is based on a combination of a clinical as well as a radiographic examination. Both components are important and often essential for an accurate diagnosis and proper treatment planning. For example, the diagnosis in cases of periodontal disease is based on a careful clinical examination to identify active periodontal disease, while radiographic examination is essential to demonstrate the past disease activity in terms of bone loss $^{1}$. 
Panoramic radiography is most often used to provide an overview of the overall bone loss and evaluate anatomical structures. However, the diagnostic quality of a panoramic radiograph is heavily dependent on careful attention to technique and processing. Furthermore, the lack of image sharpness and the superimposition of multiple structures can be considered as limitations of panoramic radiography as it may result in an underestimation of bone loss in the early stages of the disease ${ }^{1,2}$.

Whereas intra-oral radiographs are more detailed in the evaluation of bone loss levels, the use of the parallel technique is of major importance. The bisecting angle technique results in a different angle of the $X$-ray beam and may create a variable amount of bone overlap, although this technique can result in an underestimation of the disease ${ }^{3}$. In a pragmatic approach, multiple authors ${ }^{4,5,6}$ recommended taking a panoramic radiography as the first step of the periodontal assessment and the use of supplementary intra-oral radiographs to clarify dubious loci. However, clinical experience has shown that a definitive diagnosis is not always evident on a panoramic radiograph and that intra-oral radiography is very often superior. Based on this experience, it is clear that an intra-oral x-ray is preferable to a panoramic radiography for diagnosing cases of bone loss on the facial aspect of the tooth, more commonly known as buccal bone loss. However due to the 2D nature of this imaging technique, there is an unavoidable overlap of anatomical structures and a lack of 3D information ${ }^{1}$. Overlap and projection errors often lead to an over- or underestimation of the clinical situation. This is well described for the interdental bone level around teeth as well as around the implant ${ }^{7,8}$. Multiple studies evaluated diagnosis of the interdental bone level (= bone loss in between teeth) on a 2D imaging technique, although there are very few studies focusing on the facial/buccal or lingual aspect. In cases where conventional 2D radiography fails, CBCT may overcome these difficulties due to the elimination of the superimposing factor when using this technique. The use of CBCT in dentistry started in the maxillofacial field, where it was used for complex and comprehensive treatment planning. Due to the reduced radiation dose compared to a CT scan, CBCT has become a frequently used diagnostic tool in dental practice. However, published data on the accuracy of $\mathrm{CBCT}$ in the diagnosis of the buccal bone level is lacking.

This study aims to compare the accuracy of IOR as well as CBCT in the diagnosis of the extent of buccal bone loss.

\section{Material And Methods}

\section{Radiographs}

A dry skull was obtained with ethical approval and used to evaluate the accuracy of intra-oral radiography and CBCT in the diagnosis of buccal bone loss around natural teeth. The buccal bone defect was created at the most coronal level of a first premolar in the mandibula and was enlarged apically in steps of $1 \mathrm{~mm}$, starting from a defect of $1 \mathrm{~mm}$ and increasing in size up to a defect of $10 \mathrm{~mm}$ (Fig. 1). After each enlargement, an intra-oral radiograph (Digora Optimé, Soredex, Tuusula, Finland) was acquired at $70 \mathrm{kV}, 7 \mathrm{~mA}$ and $0.10 \mathrm{~s}$ using a Heliodent intra-oral x-ray tube (Sirona, Bensheim, Germany). At the same time, a CBCT (NewTom VGi, Cefla, Verona, Italy) was acquired using a field of view of $8 \times 5 \mathrm{~cm}, 110 \mathrm{kV}, 15$ 
mAs. All CBCT scans were registered with the elastix software (University Medical Center Utrecht and contributors), using the mutual information metric and a rigid transformation. Next, two observers (RP and VC) jointly selected three axial slices at different levels of the buccal bone, as well as one transverse slice. Using a macro written in ImageJ, identical slices were extracted from all other CBCT scans and saved as TIFF files. The study was conducted in accordance with the Helsinki declaration of 1975 as revised in 2000. The protocol was approved by the ethical committee of the KU Leuven Research Ethics (; NH019-2018-03-02).

\section{Observers}

Six dentists with varying specialties participated in radiographic observations, under standard viewing conditions (dimmed room, $80 \mathrm{~cm}$ distance from a HR Large format display 488 inch Samsung, Seoul, South-Korea). As it was our intention to assess inter-observer variability in the daily clinical practice, clinicians were deliberately not trained and calibrated beforehand. All measurements were performed independently by each of the observers.

\section{Assessment of bone level on the base of radiographs}

\section{Buccal defect progression}

In the first part of the study, observers received the 10 intra-oral radiographs, which registered the bone defect starting from $1 \mathrm{~mm}$ up to $10 \mathrm{~mm}$. Each observer was asked to rank the intra-oral radiographs on the extent of the buccal bone defect, starting with the smallest defect. Afterwards, they repeated the procedure with the CBCTs based on a combination of axial and transverse information.

\section{Presence or absence of a buccal defect based on CBCT}

For the second part of the study, each observer was asked to evaluate axial as well as transverse CBCT slices on the presence or absence of a buccal bone defect. To blind the observers from the nature of the investigation, the aforementioned CBCT slices were randomly pooled with a selection of axial and transverse slices, different from the first premolar on which the defect was created.

A 9-point visual analog scale (VAS) was used, in which the endpoints corresponded to absolute certainty regarding the absence or presence of a defect, respectively, and the central point corresponded to complete uncertainty regarding the presence of a defect.

\section{Statistical analysis}

The first part of the study, only containing CBCT images, was evaluated using a receiver operating curve (ROC). For the second part of the study, a weighted kappa was used to compare the true answers with those given by each observer, using linear weights. Furthermore, the absolute error in ranking the defects from 1 to 10 was calculated and compared with a hypothetical 0-value using the Wilcoxon signed rank 
tests. The significance level underwent a Bonferroni correction based on the number of observers $(n=6)$, yielding a value $\mathrm{a}=0.05 / 6 \approx 0.0833$.

\section{Case Report: clinical analysis.}

The clinical relevance of this paper can be discussed based on a clinical case of a patient who represented a recession on the right lower central incisor (Fig. 2: frontal (A) and lateral (B) view of the clinical presentation). The patient was a non-smoker in a good general health (American Society of Anesthesiologists (ASA) status 1). The patient finished orthodontic treatment some months earlier and was now referred for recession coverage of the lower incisor.

\section{Results}

\section{Buccal defect progression}

Kappa values for intra-oral radiography and CBCT are shown in Table 1. On average, kappa values were increased by 0.384 for CBCT compared with intra-oral radiography. Interestingly, three observers showed a negative kappa value for IOR, implying that their ranking of the defects was worse than random scoring $(\mathrm{K}=0)$. Apart from two observers, who showed equal kappa values for both modalities, CBCT showed superior defect ranking. The average error in ranking as well as the results of Wilcoxon signed rank tests for each observer are shown in Table 2. For IOR, four out of six observers showed a significant ranking error, whereas none of the observers showed a significant error for СВCT. The percentage of rankings that were within 1 of the true rank was $32 \%$ for IOR and $42 \%$ for CBCT.

Table 1

Kappa value for each observer for intra-oral radiography (IOR) and cone-beam computed tomography (CBCT)

\begin{tabular}{|lll|}
\hline Observer & IOR vs. ground truth & CBCT vs. ground truth \\
\hline General Dentist & -0.273 & 0.212 \\
\hline Oral surgeon & -0.152 & 0.818 \\
\hline Periodontist & 0.394 & 0.394 \\
Endodontist & 0.515 & 0.515 \\
Implant surgeon & -0.333 & 0.333 \\
Radiologist & 0.212 & 0.394 \\
Average & 0.061 & 0.444 \\
\hline
\end{tabular}


Table 2

Average error from the true rank of defect severity for intra-oral radiography (IOR) and cone-beam computed tomography (CBCT), and percentage of rankings within 1 of the true rank. Asterisks $(*)$ denote a significant average error.

\begin{tabular}{|lllll|}
\hline Observer & IOR & \multicolumn{3}{l|}{ CBCT } \\
\hline & Avg. error & $\%$ with error $\leq 1$ & Avg. error & $\%$ with error $\leq 1$ \\
\hline General Dentist & 4.2 & $10 \%$ & 2.6 & $30 \%$ \\
\hline Oral surgeon & $3.8^{*}$ & $30 \%$ & 0.6 & $80 \%$ \\
\hline Periodontist & $2.0^{\star}$ & $60 \%$ & 2.0 & $30 \%$ \\
\hline Endodontist & 1.6 & $60 \%$ & 1.6 & $40 \%$ \\
\hline Implant surgeon & $4.4^{*}$ & $0 \%$ & 2.2 & $30 \%$ \\
\hline Radiologist & $2.6^{*}$ & $30 \%$ & 2.0 & $40 \%$ \\
\hline Average & 3.1 & $32 \%$ & 1.8 & $42 \%$ \\
\hline
\end{tabular}

\section{Presence or absence of a buccal bone defect based on CBCT}

The average area under the curve (AUC) of the ROC was 0.892 for all observers (Fig. 3). Detailed information of the area under the curve for each observer is found in Table 3. Three observers showed an AUC above 0.95, whereas the other observers' AUC ranged between 0.67 and 0.89 (Fig. 4).

Table 3

Receiver operating characteristic area under the curve (AUC), sensitivity and specificity for each observer, along with average confidence according to VAS

\begin{tabular}{|lllll|}
\hline Observer & AUC & Sensitivity & Specificity & Average confidence (\%) \\
\hline General Dentist & 0.848 & 0.82 & 0.77 & 68 \\
\hline Oral surgeon & 0.665 & 0.82 & 0.62 & 95 \\
\hline Periodontist & 0.966 & 0.94 & 0.92 & 73 \\
\hline Endodontist & 0.959 & 0.88 & 0.92 & 53 \\
\hline Implant surgeon & 0.986 & 0.94 & 0.85 & 85 \\
\hline Radiologist & 0.892 & 0.94 & 1.00 & 94 \\
\hline
\end{tabular}

The overall sensitivity and specificity of CBCT in the diagnosis of the presence or absence of a buccal bone defect was 0.89 and 0.85 , respectively. On average, observers had $78 \%$ confidence in their diagnosis; an increased confidence was not related to an increased diagnostic performance. 


\section{Case Report: clinical analysis.}

Based on the intra-oral radiograph (Fig. 5) of the lower incisors of the patient, no major problem was expected. The interdental bone peaks were within the natural range and apart from a slight wider periodontal ligament, this radiograph did not seem to report a disturbing situation. Signs to suggest that the buccal bone around the right lower incisor was absent for more than $2 / 3$ of the root length were absent. CBCT images (Planmeca, Helsinki, Finland) of the same area reveal unexpected problems with buccal bone loss, unable to be diagnosed on the 2D radiograph (Fig. 6). Indeed, while no major problem in terms of absence of buccal bone was expected on the intra-oral radiograph, CBCT clearly illustrated a doubtful prognosis of the right central incisor, with this tooth being for less than $1 / 3$ anchored in the bone.

\section{Discussion}

The current study, along with the case presentation, clearly illustrates the impact of overlapping anatomical structures on an intra-oral radiograph that may lead to a significant radiographic underestimation of the bone level. In the literature overlap of anatomical structures and image distortion are well described shortcomings of intra-oral radiographs such as peri-apical radiographs ${ }^{9}$. Especially for diagnosis of a buccal bone defect, volumetric images with high dimensional accuracy and no overlap of buccal and lingual/palatal bone are required. CBCT provides 3D information at a high resolution of teeth, surrounding bone and other anatomical structures ${ }^{10,11}$. In implant dentistry, CBCT seems to be the preferred pre-operative radiographic method due to its high dimensional accuracy ${ }^{12,13,14}$. Keeping those advantages in mind, CBCT provides opportunities to more accurate diagnosis in the dental field.

To the best of our knowledge, there is no literature available discussing the accuracy of IOR and CBCT to diagnose buccal bone defects. The results of the present study describe that the interpretability of buccal bone defects differs between IOR and CBCT. The overall reliability for diagnosis of a buccal bone defect was low for IOR and high for CBCT. In other words, it is far more likely to underestimate a buccal bone defect with IOR compared to CBCT.

While the diagnostic benefit of CBCT over 2D radiography is demonstrable, one should also consider the radiation risk ${ }^{15}$. An additional consideration is the wide range in image quality and radiation dose between CBCT machines, or between different exposure protocols of a CBCT unit. The imaging performance of $\mathrm{CBCT}$ is dictated by several factors, including but not limited to $\mathrm{kV}, \mathrm{mAs}$, and voxel size, which are not standardized between manufacturers or clinics ${ }^{15,16,17}$.

The use of a cadaver can be seen as a limitation of the study, although it allowed for a ROC analysis due to the availability of the ground truth. Furthermore, creating extensive bone defects and taking multiple intra-oral radiographs and CBCT scans in a patient would be ethically unacceptable. 
Overall, one should always keep in mind the principles of justification and optimization, with a need to go for indication-oriented and patient-specific imaging should always be kept in mind (ALARA/ALADAIP principle) ${ }^{18,19}$. Considering the ongoing evolution in CBCT imaging (e.g. lower radiation dose, shorter acquisition time, etc.) further studies focusing on CBCT for radiographic bone level assessment should be conducted.

\section{Conclusion}

When СВCT images are present for justified indications other than bone level assessment, the available $\mathrm{CBCT}$ images are more accurate than 2D images to assess buccal periodontal bone and thus preferred. CBCT should be used as a secondary radiographic tool; for example, in cases with clinical doubts or lacking information of the buccal bone level, an indication-specific CBCT can also be justified to avoid improper treatment planning and complications at outcome. For other clinical applications, intra-oral radiography remains the standard method for radiographic evaluation.

\section{Declarations}

\section{Ethics approval:}

The study was ethically approved by Katholieke Universiteit Leuven Research Ethics, reference number: NH019-2018-03-02.

\section{Informed Consent:}

was obtained for use of the clinical case.

\section{Consent for publication:}

Not applicable.

\section{Availability of data and materials:}

The dataset used and analysed during the current study are available from the corresponding author on reasonable request.

\section{Competing interests:}

Véronique Christiaens: The author would like to report on a collaboration agreement with Southern Implants (Irene, South-Africa). Rest of the authors does not have any competing interest. 


\section{Funding:}

Ruben Pauwels is supported by the European Union Horizon 2020 Research and Innovation Programme under the Marie Skłodowska-Curie grant agreement number 754513 and by the Aarhus University Research Foundation (AIAS-COFUND).

\section{Authors' contributions:}

Véronique Christiaens: Data collection, first and final draft

Bassant Mowafey: Data collection, figures, first draft

Ruben Pauwels: Statistical analysis, figures, first and final draft

Reinhilde Jacobs: Structure, first and final draft

\section{Acknowledgements:}

Not applicable

\section{References}

1. Rushton VE, Horner K. The use of panoramic radiology in dental practice. J Dent . 1996; 24:185-201.

2. Akesson L, Hakansson J, Rohlin M. Comparison of panoramic and intraoral radiography and pocket probing for the measurement of the marginal bone level. J Clin Peridontol 1992; 19:326-332.

3. Lang NP, Hill RW. Radiographs in periodontics. J Clin Periodontol 1977; 4:16-28.

4. Akesson L, Rohlin M, Hakansson J. Marginal bone in periodontal disease: an evaluation of image quality in panoramic and intraoral radiography. Dentomaxillofac Radio 1989; 18:105-112.

5. Molander B, Ahlqwist M, Grondahl H, Hollen-der L. Agreement between panoramic and intraoral radiography in the assessment of marginal bone height. Dentomaxillofac Radio 1991; 20:155-160.

6. Hirschmann PN, Horner K, Rushton VE. Selection criteria for periodontal radiography. Br Dent J 1994; 176:324-325.

7. Christiaens V, Jacobs R, Dierens M, Vervaeke S, De Bruyn H, Cosyn J. Intra-oral radiography lacks accuracy for the assessment of peri-implant bone level affected by advanced peri-implantitis a controlled study. Eur J Oral Implantol 2017; 10:435-441.

8. Christiaens V, De Bruyn H, Thevissen E, Koole S, Dierens M, Cosyn J. Assessment of periodontal bone level revistied: A controlled study on the diagnostic accuracy of clinical evaluation methods and periapical radiography. Clin Oral Investig 2018; 22:425-431.

9. Al Ohski A, Paulsson L, Rohlin M, Ebrahim E, Lindh C. Measurability and reliability of assessments of root length and mariganl bone level in cone beam CT and intraoral radiography: a study of adolescents. Dentomaxillofac Radiol. 2019; 48:20180368. 
10. Hans MG, Palomo JM, Valiathan M. History of imaging in orthodontics from Broadbent to conebeam computed tomography. Am J Orthod Dentofacial Orthop 2015; 148:914-821.

11. Dief S, Veitz-Keenan A, Amintavakoli N, McGowan R. A systematic review on incidental findings in cone beam computed tomography (CBCT) scans. Dentomaxillofac Radiol 2019; 48:20180396.

12. Angelopoulos C, Aghaloo T. Imaging technology in implant diagnosis. Dent Clin North Am 2001; 55:141-158.

13. Kamburoglu K, Kilic C, Ozen T, Yuksel SP. Measurements of mandibular canal region abotained by cone beam computed tomography: a cadaveric study. Oral Surg Oral Med Oral Pathol Oral Radiol Endod 2009; 107:34-42.

14. Hassan B, van der Stelt P, Sanderink G. Accuracy of three-dimensional measurements obtained from cone beam computed tomography surface-rendered images for cephalometric analysis: influence of patient scanning position. Eur J Orthod 2009; 31:129-134.

15. Beganovic A, Ciraj-Bjelac O, Dyakov I, Gershan V, Kralik I, Milatovic A, Salat D, Stepanyan K, Vladimirov A, Vassileva J. IAEA survey of dental cone beam computed tomography practice and related patient exposure in nine Central and Eastern European countries. Dentomaxillofac Radiol 2020

16. Pauwels R, Beinsberger J, Stamatakis H, Tsiklakis K, Walker A, Bosmans H, Bogaerts R, Jacobs R, Horner K; SEDENTEXCT Project Consortium. Comparison of spatial and contrast resolution for conebeam computed tomography scanners. Oral Surg Oral Med Oral Pathol Oral Radiol. 2012;114:127-35

17. Pauwels R, Araki K, Siewerdsen JH, Thongvigitmanee SS. Technical aspects of dental CBCT: state of the art. Dentomaxillofac Radio. 2015; 44:20140224. doi: 10.1259/dmfr.20140224.

18. Pauwels R. Cone-beam CT for dental and maxillofacial imaging: Dose matters. Radiat Prot Dosimetry 2015; 165: 156-161.

19. Oenning AC, Jacobs R, Pauwels R, Stratis A, Hedesiu M, Salmon B; DIMITRA Research Group. Conebeam CT in paediatric dentistry: DIMITRA project position statement. Pediatr RAdiol. 2018; 48:308316. doi: 10.1007/s00247-017-4012-9.

\section{Figures}



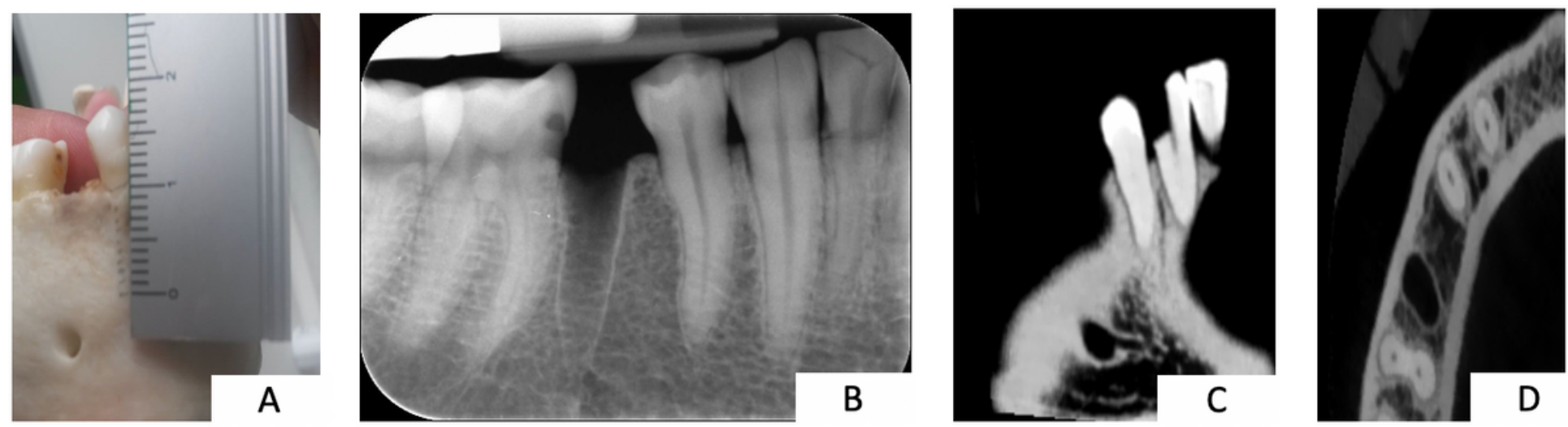

$1 \mathrm{~mm}$
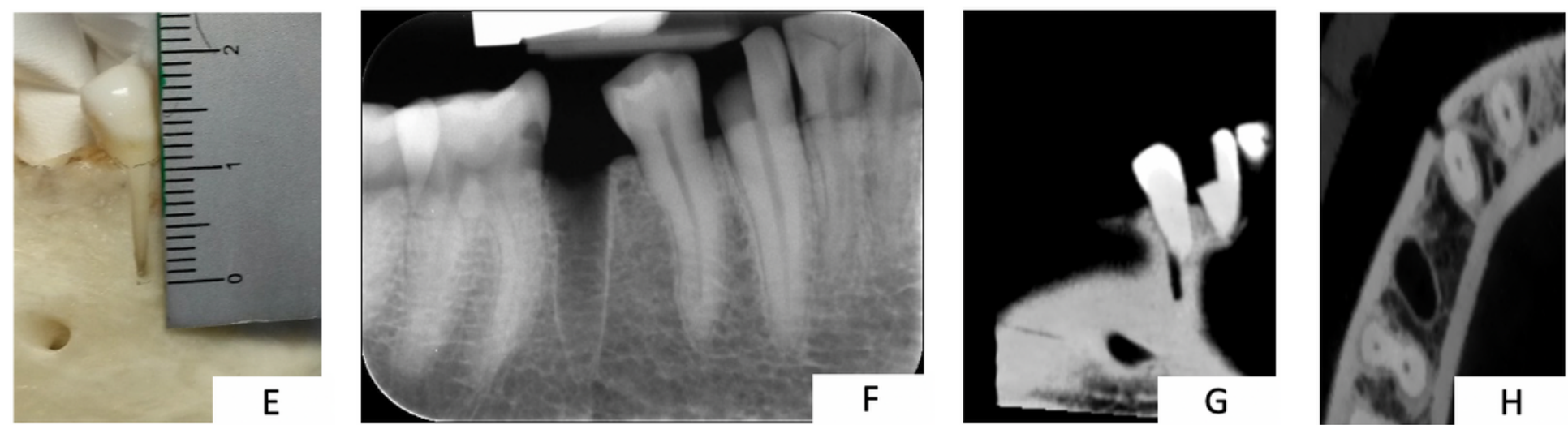

$10 \mathrm{~mm}$

Figure 1

The buccal bone defect was created at the most coronal level of a first premolar in the mandibula and was enlarged apically in steps of $1 \mathrm{~mm}$. Buccal defect of $1 \mathrm{~mm}$ created (A), peri-apical radiography (B) and $C B C T(C+D)$ of the $1 \mathrm{~mm}$ defect. Buccal defect of $10 \mathrm{~mm}$ created $(E)$, peri-apical radiography $(F)$ and CBCT $(\mathrm{G}+\mathrm{H})$ of the $10 \mathrm{~mm}$ defect. 

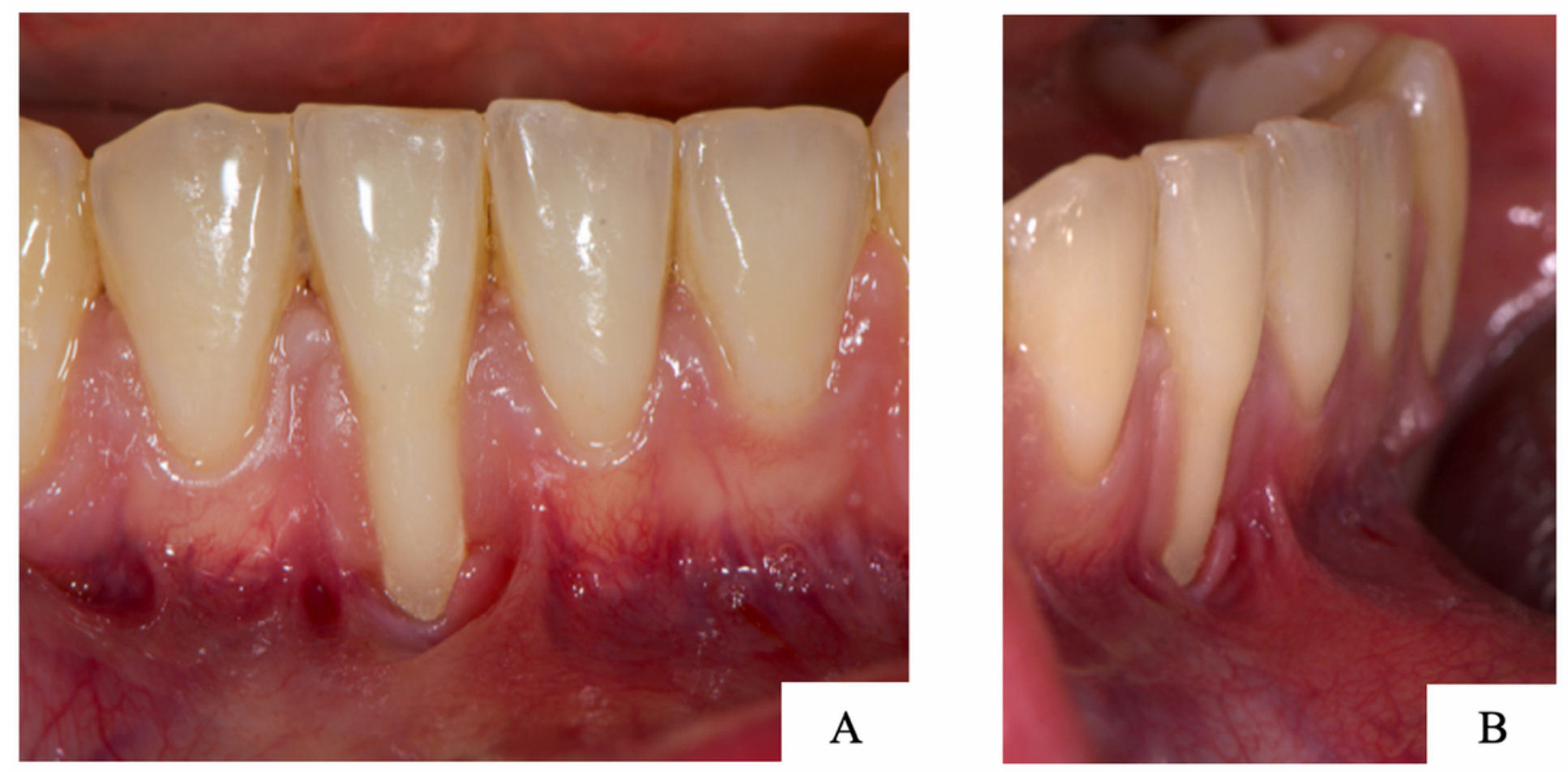

Figure 2

Frontal (A) and lateral view (B) of the lower incisors with a recession on the right central incisor.

\section{All observers $(A U C=0.892)$}

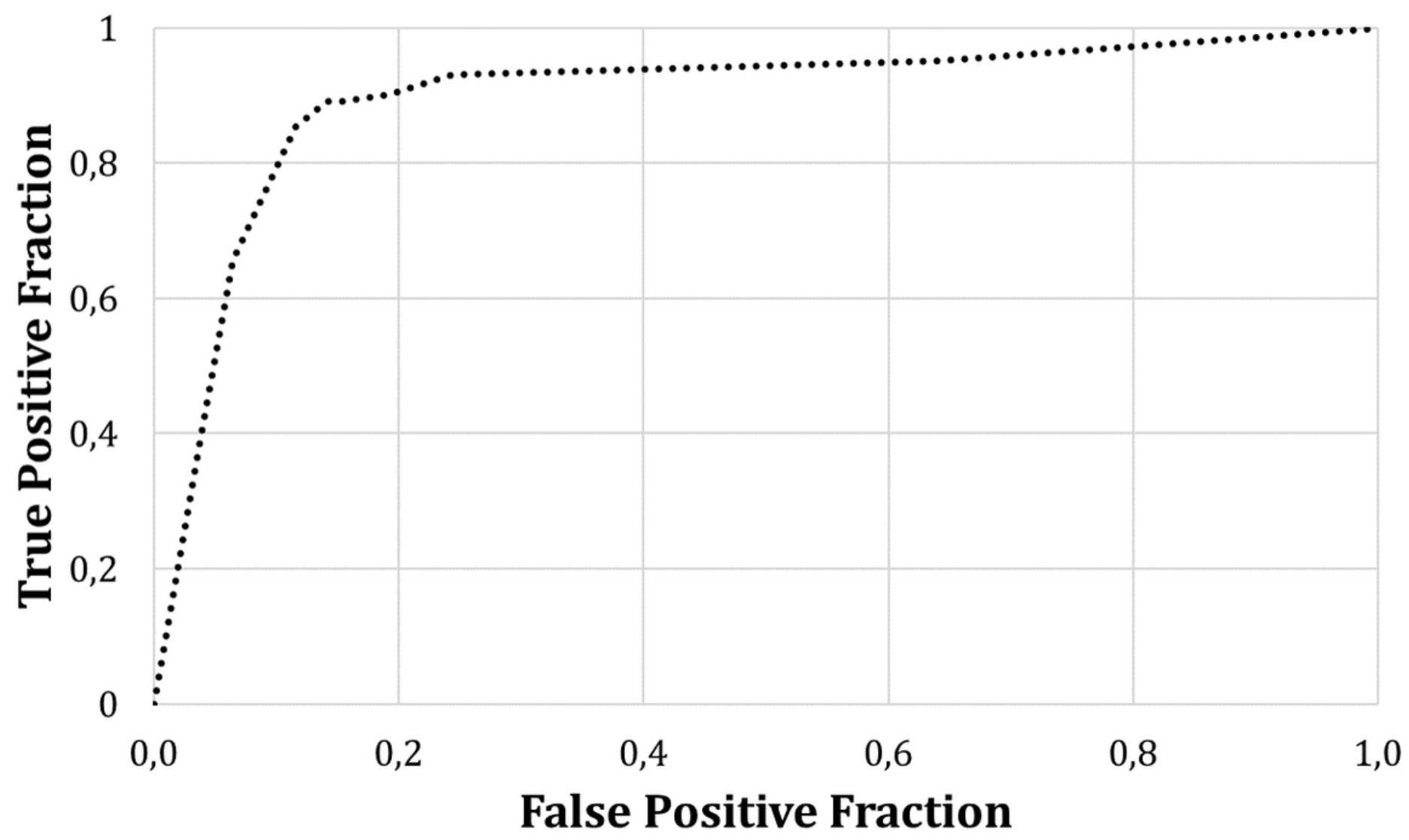




\section{Figure 3}

Receiver operating characteristic curve for cone beam computed tomography analysis of buccal bone defects, averaged over all observers. AUC = area under the curve.
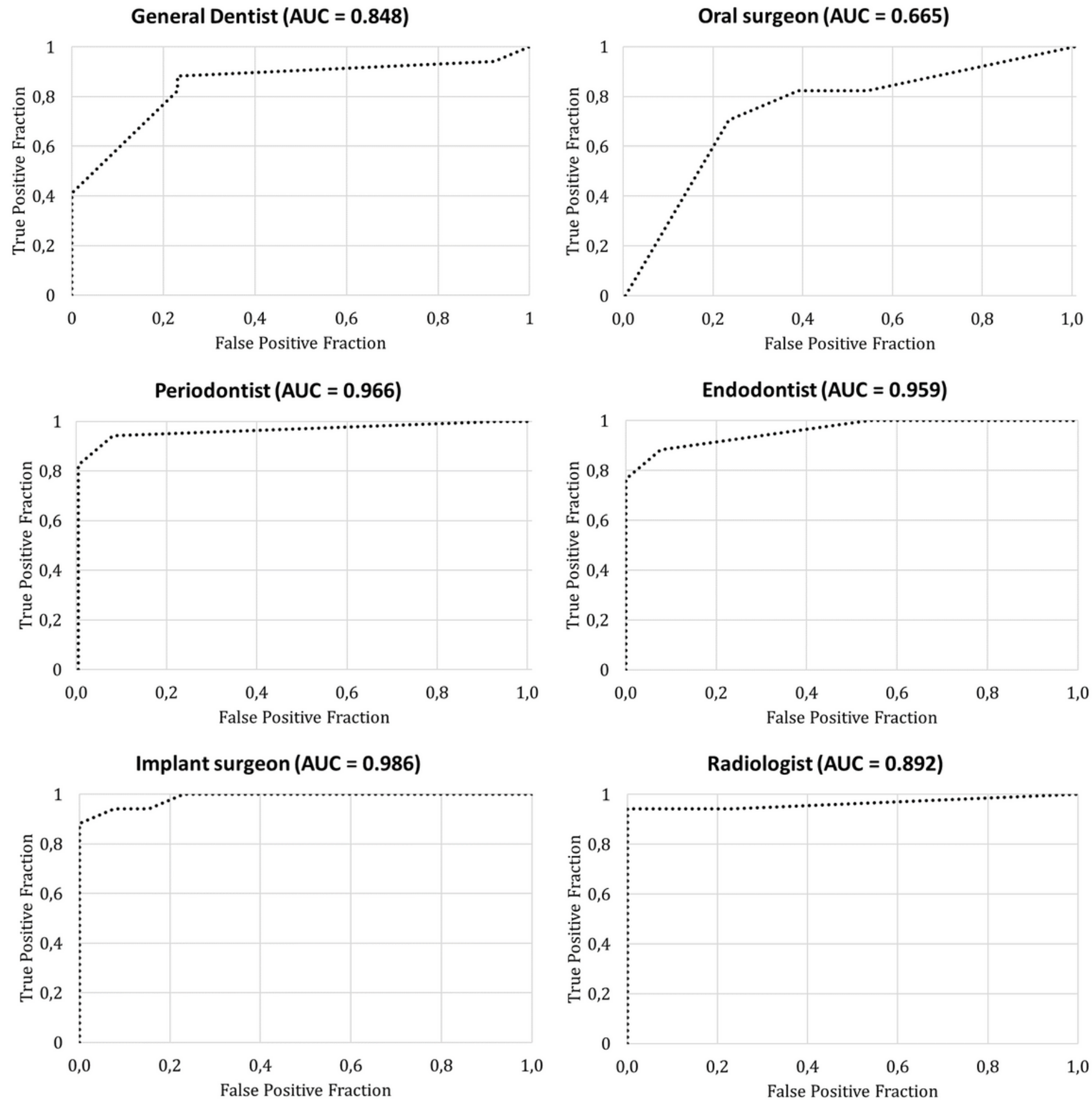

Figure 4

Receiver operating characteristic curve for cone beam computed tomography analysis of buccal bone defects, for individual observers. AUC = area under the curve. 


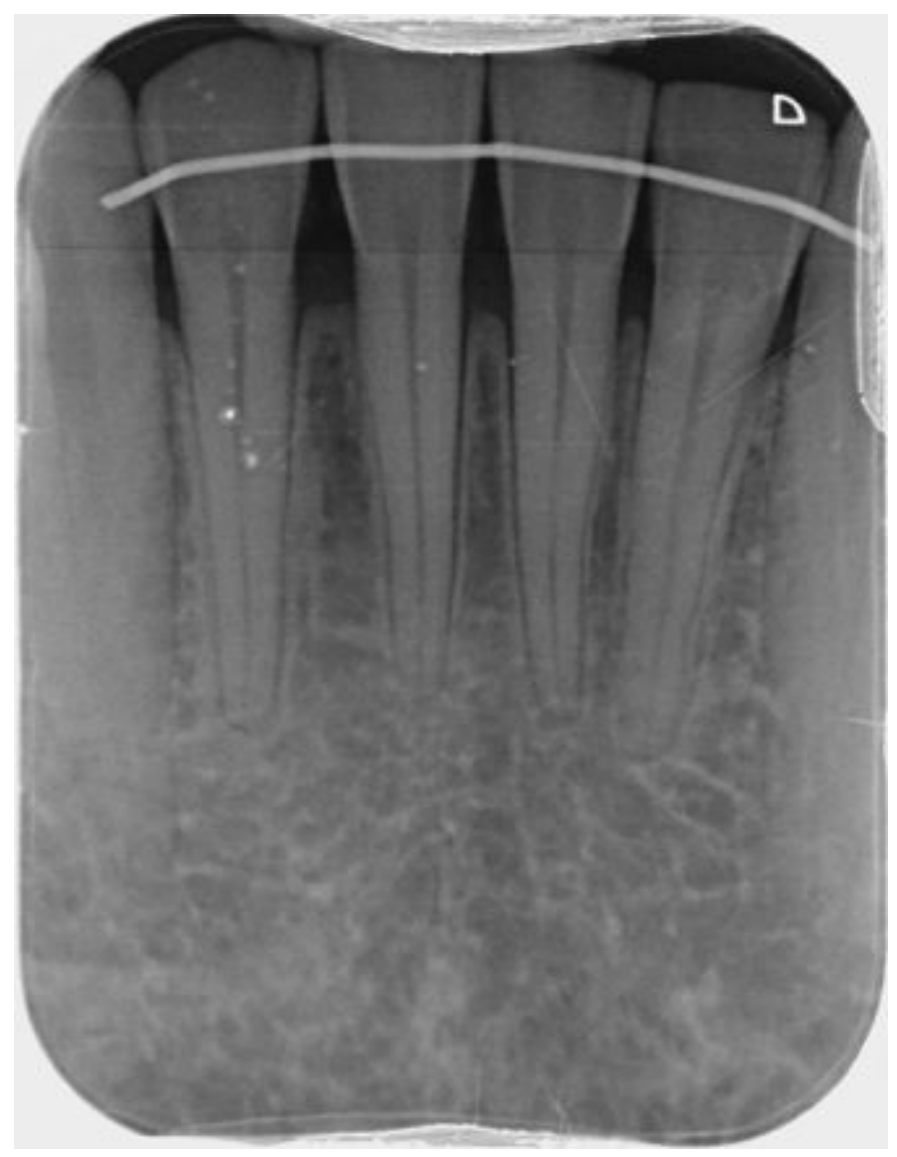

Figure 5

Intra-oral radiograph of the lower incisors.

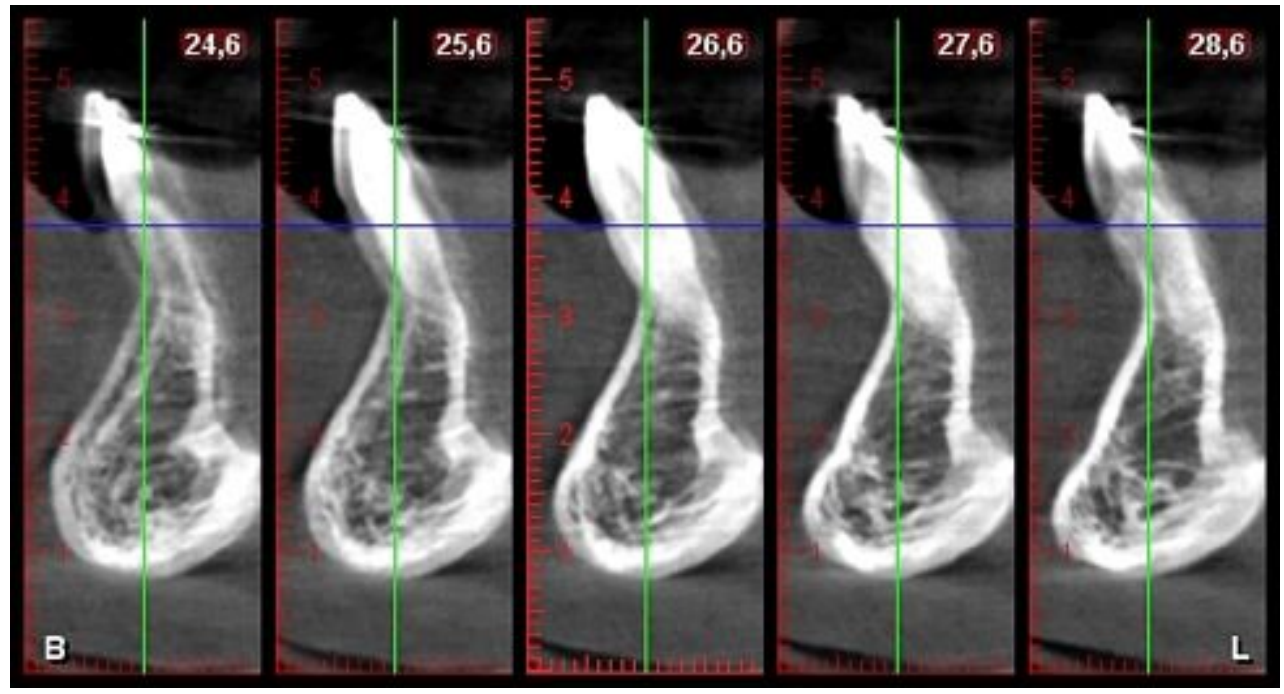

Figure 6

CBCT cross-sectional image of the right lower incisor. 